\title{
Supporting Information: \\ Effective negative diffusion of singlet excitons in organic semiconductors
}

\section{Contents}

SI 1 - Transient Absorption Microscopy.

SI 2 - Probe scan of 1951 USAF resolution test target with TAM ...................................................... 2

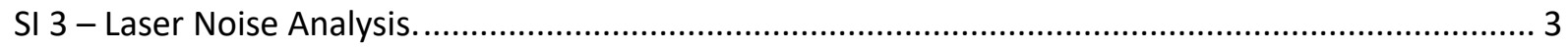

SI 4 - Sensitivity and spatial precision of the transient absorption microscope. .................................. 4

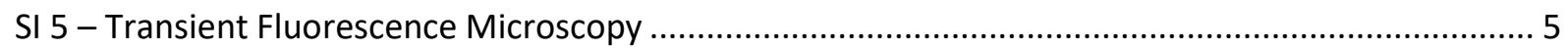

SI 6 - Determination of the point spread function of the confocal microscope................................... 6

SI 7 - Spatial precision of the Transient fluorescence microscope...................................................... 7

SI 8 - Fabrication and characterization of the tetracene crystals.................................................... 8

SI 9 - Extended analysis of anisotropic triplet exciton diffusion in tetracene...................................... 9

SI 10 - Comparison of Gaussian fit and averaging for FWHM extraction.......................................... 10

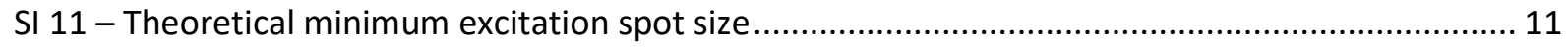

SI 12 - Spatial transient absorption measurements in tetracene..................................................... 12

SI 13 - Intensity dependent measurements of tetracene with simplified model................................. 13

SI 14 - Differential equation for the simulation of the effective negative exciton diffusion ............... 14

SI 15 - Measurement and simulation of time resolved fluorescent decay.......................................... 15

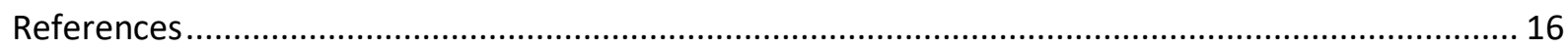




\section{SI 1 - Transient Absorption Microscopy}

The pump probe measurements were performed with a $\lambda=820 \mathrm{~nm}$ pulsed laser beam from a Ti:Sapph oscillator (MaiTai, $80 \mathrm{Mhz}$ ) as probe and its second harmonic at $\lambda=410 \mathrm{~nm}$ as pump. A probe at 820 $\mathrm{nm}$ for tetracene corresponds to photo-induced absorption of the triplet state ${ }^{1,2}$. The repetition rate of both pulses was reduced to $100 \mathrm{kHz}$ by a pulse picker (APE pulseSelect Dual), followed by chopping the pump beam down to $50 \mathrm{kHz}$ using an acousto-optic modulator (Gooch and Housego, I-M1102C10B6-3-GH26). The time resolution was limited by the duration of the laser pulses at the sample position, which was measured to be $200 \mathrm{fs}$ (see SI Fig. S4). The probe beam was sent through a delay stage with 2 ns traveling length (NewPort) and XY-galvo scanners (Thorlabs, GVS012), both controlled by a data acquisition card (NI-DAQ USB-6366) $)^{3}$. The step size of the galvo scanners was set to $2 \mathrm{mV}$, corresponding to $118 \mathrm{~nm}$ per pixel. The pump beam was kept at a fixed position while the probe was scanned. Both beams were merged through a dichroic mirror and focused on the sample with a NA 0.95 objective (Leica apochromat 100x). The transmitted light was collected with a 0.85 NA objective (Nikon Plan LWD 100x). Two triggered photodiodes were used to detect the maxima of the probe pulse intensity before and after the sample for balanced detection ${ }^{4}$. For long integration times of $8 \times 10^{4}$ pump pulses (=1.6 second) per pixel, a sensitivity of $\frac{\Delta T}{T} \sim 2 \times 10^{-6}$ was achieved (see SI Fig. S4). Reliable measurements with spatial precision of $\sim 30 \mathrm{~nm}$ (SI Fig. S4) are performed down to peak signals of $10^{-4}$, which corresponds to excitation densities $\sim 2 \times 10^{18} \mathrm{~cm}^{-3} \mathrm{~s}^{-1}$ when probing at $820 \mathrm{~nm}$. The minimum peak-intensity is much higher than the sensitivity because for a complete measurement much lower transient signals must be measured away from the center of the Gaussian and at longer pump-probe delay times. 

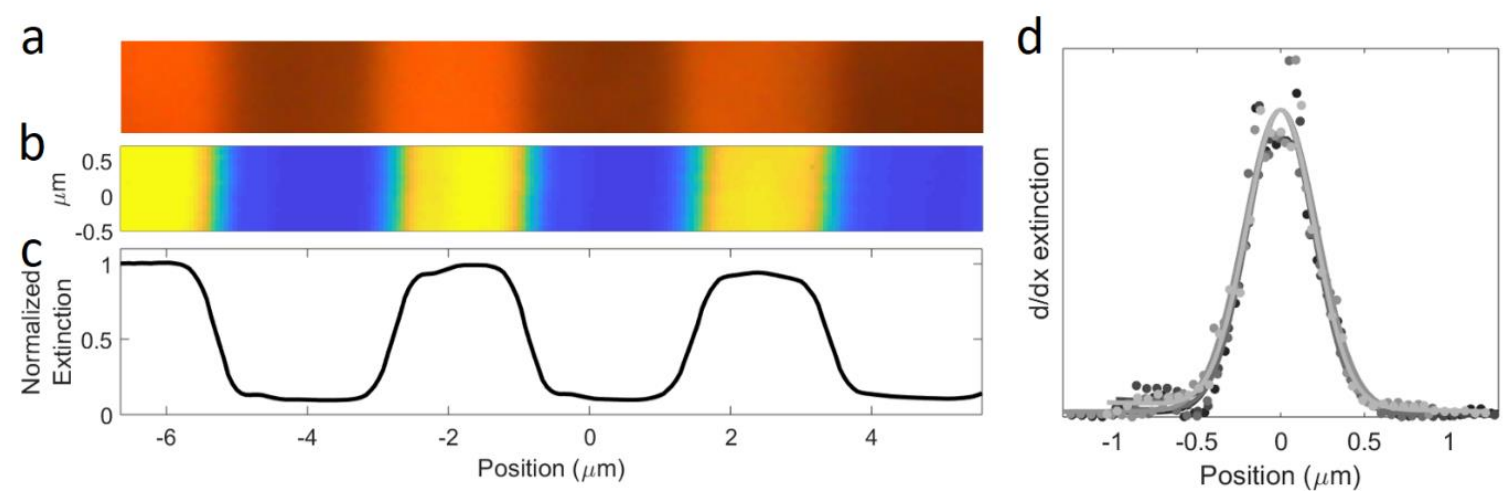

Figure S2: To calibrate the transient TAM and to determine the spatial resolution of the probe beam $(\lambda=820 \mathrm{~nm})$ in the microscope a test measurement without the pump was performed using a Leica objective (100x NA=0.95 apochromat). The test sample is a 1951 USAF resolution test target, with as target object group 7, element 6 , with $2.19 \mu \mathrm{m}$ per line. (a) shows a widefield reflection measurement, (b) shows the extinction map (1- transmission) of the same region of the target by scanning the probe across the target. (c) Shows a horizontal line scan of (b). The theoretical spatial resolution is defined by the diffraction limit: Res $=\lambda /(2 \cdot N A)=432 \mathrm{~nm}$. In (d) we plot the absolute value of the derivative of the extinction with respect to position for the 5 edges in Figs. (b) and (c). We obtain the resolution of the measurement by fitting the derivatives with a Gaussian function to obtain a full-width at halfmaximum of $460 \pm 5 \mathrm{~nm}$. This value is within $10 \%$ of the diffraction limit and is used as convolution for the simulations in Fig. 4 of the manuscript. 

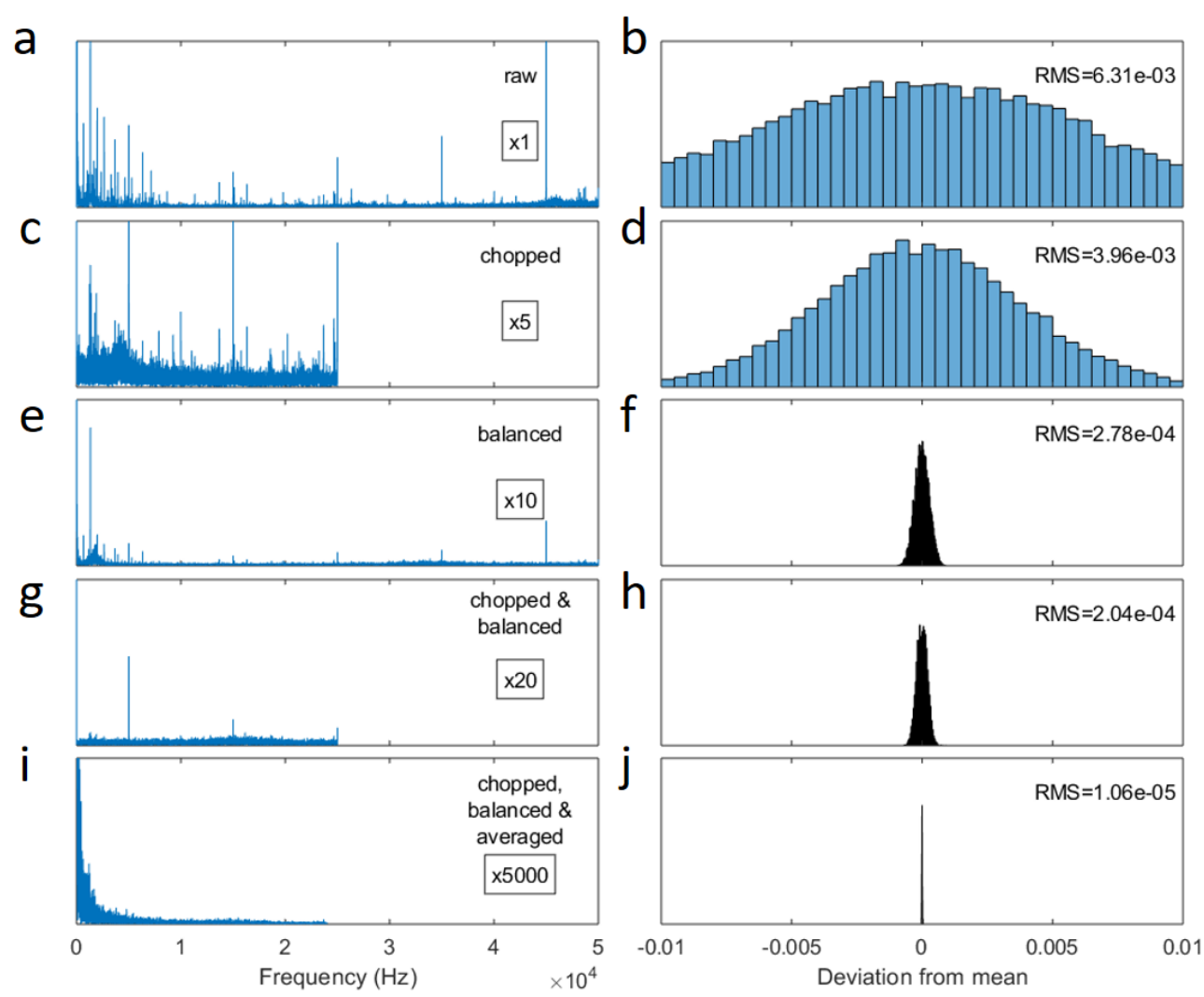

Figure S3: The spatial sensitivity of the microscope is not only limited by the diffraction limit, but also by the signal to noise of the measured exciton distribution profiles. For the TAM, the signal to noise depends on the laser stability, stability of the laser pointing (including the galvo-mirrors) and the detection electronics. Since the noise in the laser and detection is usually larger than the signal $(\Delta T)$, an averaging method is required to extract any useful information from the data. This is often done by using a Lock-In amplifier, but we employ here a technique described by Werley et al. ${ }^{4}$ using a National Instruments Digital-Analogue converter (NI-DAQ) to acquire the intensity of individual laser pulses followed by digital filtering of the signals. This method has the advantage that it may decrease the 'dwell time' between different data points and allows for a full quantification of the noise. ${ }^{4}$ In this figure, we show the analysis of $10^{5}$ laser pulses ( 1 second acquisition time at $100 \mathrm{kHz}$ ). The figure shows how the laser noise reduces after each processing step. For each processing step, the Fourier transform of the data is shown on the left, and the deviation of each pulse from the mean is shown on the right. The number in the box in all the left figures is the relative values of the $y$-axis, normalized to the $y$ axis of Fig. (a). The root mean square (RMS) of the signal after every processing step is given in every figure in the right column. ( $a$ and $b$ ) show the raw data i.e. the measured peak intensity of $10^{5}$ laser pulses. In ( $c$ and $d$ ) each pulse is divided by the subsequent pulse, filtering out low frequency noise and mimicking chopping in a transient measurement. (e and f) show that balanced detection (dividing each pulse intensity by the intensity of the same laser pulse measured by a different photodiode) results in an order of magnitude improvement of the signal to noise ratio, which is further reduced in ( $\mathrm{g}$ and $\mathrm{h}$ ) by combining the chopping and the balanced detection. Finally, we can improve the signal to noise ratio significantly when we average over each 500 datapoints $(5 \mathrm{~ms})$, resulting in a standard deviation (RMS) of $1.06 \times 10^{-5}$ ( $i$ and $\left.j\right)$. 
SI 4-Sensitivity and spatial precision of the transient absorption microscope.
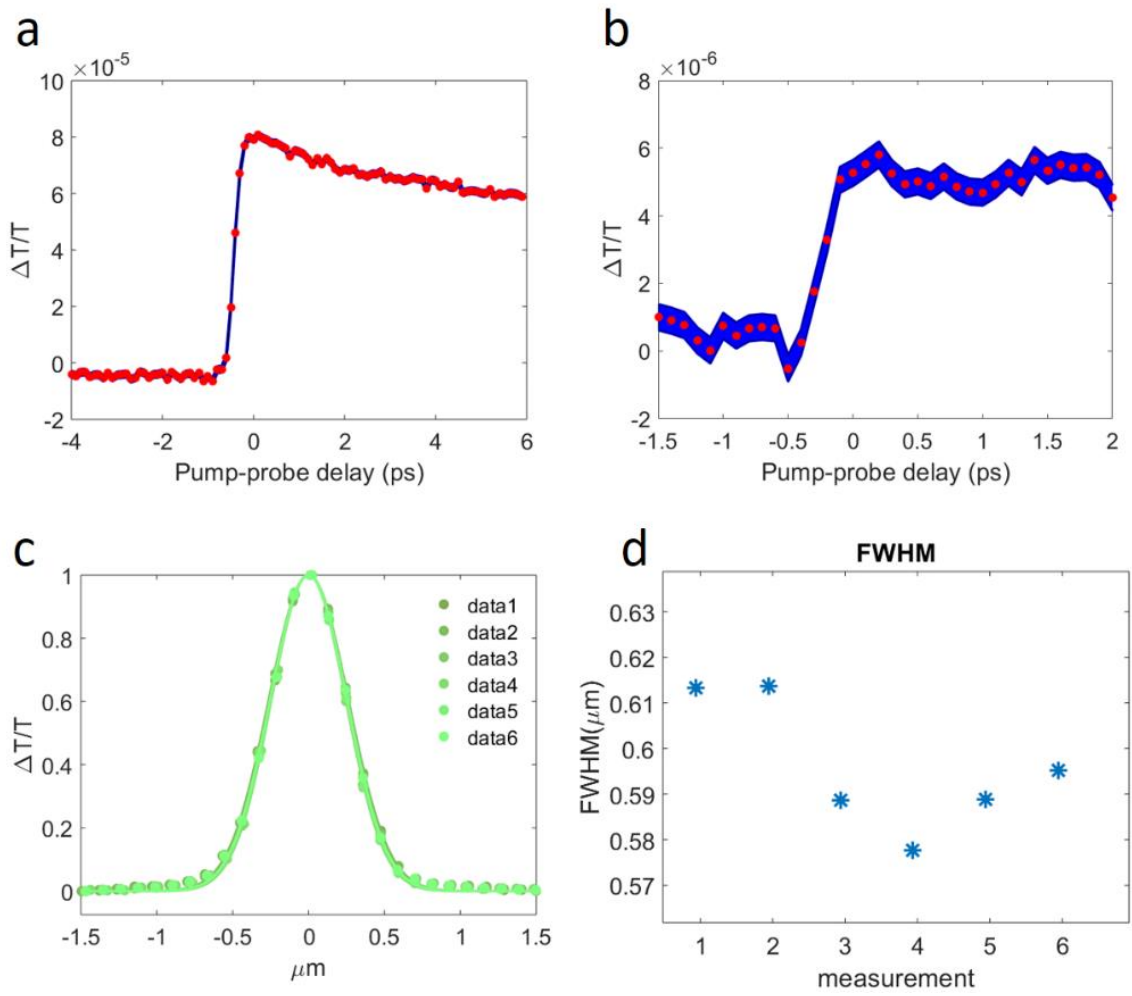

Figure S4: In the previous section we have determined the laser noise, it is however not trivial to get the sensitivity for practical measurements from this data, because of other factors such as the stability and repeatability of the galvo scanners and laser pointing. To determine the sensitivity of the TAM, i.e., the minimum differential transmission that can be measured, we performed measurements at low excitation powers, shown in Figs (a) and (b) for 30 and $3 \mathrm{fJ} /$ pulse respectively. The measurement is done with the transient setup described in the manuscript, but without scanning the probe laser. The sample is a tetracene crystal. Each data point is integrated for 1.6 seconds, which corresponds to $8 \mathrm{x}$ $10^{4}$ pulse pairs. The red data points show the average of these datapoints and the blue shaded area corresponds to the standard error of the mean $(=$ standard deviation $/ \sqrt{N})$. From these measurements the minimum sensitivity is estimated to be $2 \times 10^{-6}$. To obtain the spatial precision, we measured the laser induced singlet distribution in a tetracene crystal multiple times at the same pumpprobe delay. The (almost overlapping) cross sections of these scans are shown in (c) and the FWHM of these distributions are shown in (d). From the standard deviation of the measured FWHM, we estimate a spatial precision of $\pm 15 \mathrm{~nm}$. 
Tetracene crystals were excited in a Nikon Ti-E microscope with a 100x objective (Nikon TU Plan Fluor). The excitation laser $(\lambda=470 \mathrm{~nm}$ ) was the second harmonic of the output of a Ti:Sapph oscillator (MaiTai, $80 \mathrm{Mhz}$ ) that was down sampled to $500 \mathrm{kHz}$ using an pulse picker (APE pulseSelect). The fluorescence was collected in reflection and sent to a confocal scanning head (Nikon C2, $30 \mu \mathrm{m}$ pinhole) connected to a time-correlated single photon counting detector (Picoquant, TimeHarp 300), with an instrument response of $50 \mathrm{ps}$ defining the time resolution. The temporal range of the measurements was limited by the electronics of the system and the software (SymphoTime 64) to a maximum of $2 \mu \mathrm{s}$. The laser reflection is filtered by a dichroic mirror ( $447 \mathrm{~nm}$ edge) and a $535 \pm 25 \mathrm{~nm}$ band-pass filter. The spatial sensitivity was estimated at $10 \mathrm{~nm}$ (see SI Fig. S9). 
SI 6-Determination of the point spread function of the confocal microscope.

a
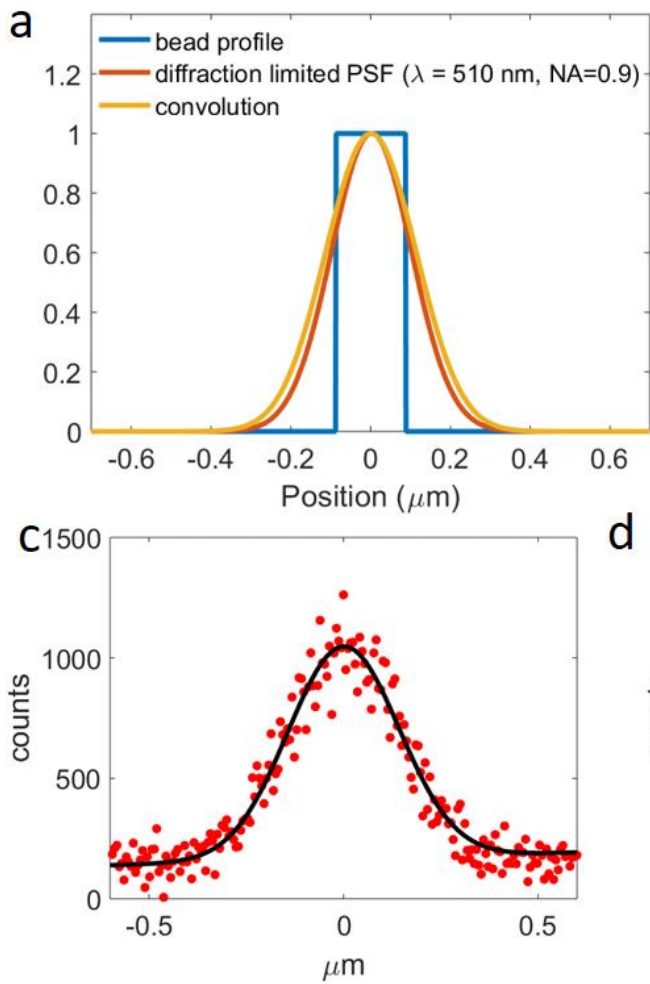

b

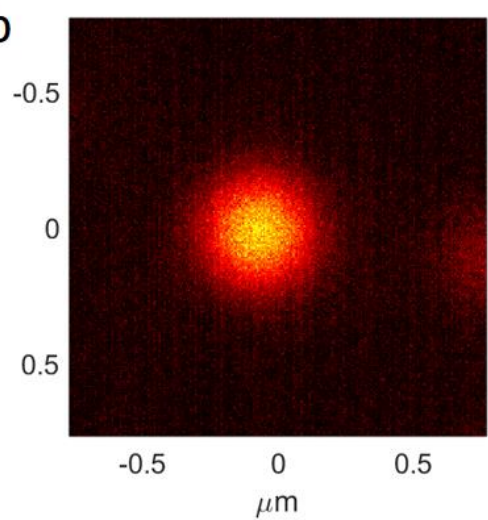

d

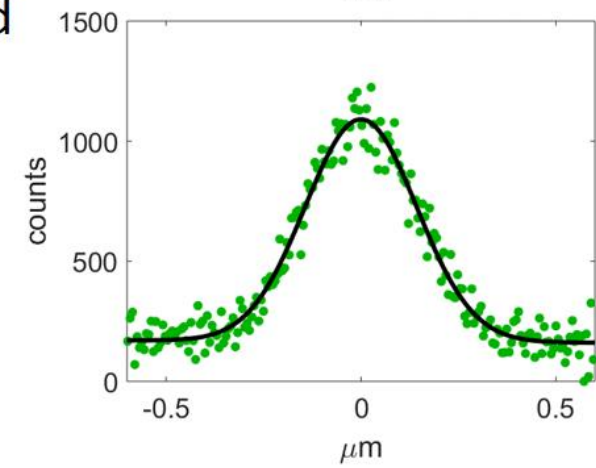

Figure S6: The resolution of a fluorescence measurement of a laser induced excitation spot is the convolution of the excitation spot size with the point spread function (PSF) of the microscope. To determine the resolution of the detection, we have measured the emission of a fluorescent bead with a $175 \mathrm{~nm}$ diameter (PS-Speck, Green fluorescent microspheres (Component B), 505/515 nm excitation/emission maxima) under wide field UV illumination with a 0.9 NA objective. The diffraction limited resolution of a perfect point source emitting at $510 \mathrm{~nm}$ with an 0.9 NA objective would give a PSF of $\lambda /\left(2^{*} N A\right)=286 \mathrm{~nm}$ (orange curve in (a)). A diffraction limited detection of the bead is a convolution of this PSF and the profile of the bead (blue curve in (a), which gives the yellow curve with a FWHM of $330 \mathrm{~nm}$ ). The image of the measurement of the fluorescence from the bead is shown in (b) and the horizontal and vertical cross sections in (c) and (d), respectively. The FWHM of these profiles are $337 \mathrm{~nm}$ and $338 \mathrm{~nm}$, which are very close to the theoretical minimum of $330 \mathrm{~nm}$. This PSF with a FWHM of $337 \mathrm{~nm}$ is used as convolution for the simulation in Fig. 4 of the manuscript. 
SI 7 -Spatial precision of the Transient fluorescence microscope.
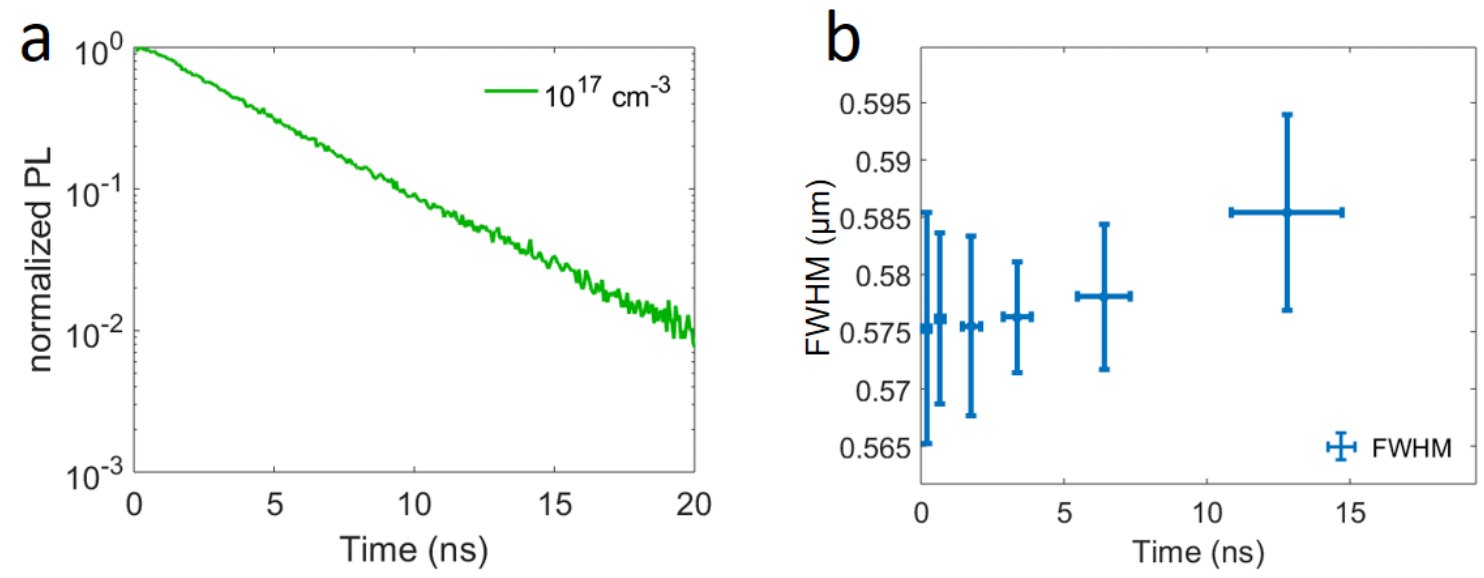

Figure S7: The size of the laser excitation is fundamentally limited by the diffraction limit. However, changes in the spatial profile of this initial excitation can be measured with a much higher precision. The precision is not determined by the diffraction limit, but rather by the signal to noise of the gaussian fluorescence profiles ${ }^{2,5}$. To estimate the minimum change that we can measure, we used a sample of a dye in a PMMA matrix. The dye, Lumogen Green, emits at a similar wavelength as the singlet excitons in tetracene. By taking a low dye concentration ( $\sim \% / \mathrm{wt})$, we suppress exciton-exciton annihilation and diffusion of excitons to other molecules, which enables the determination of the spatial precision of the microscope to measure the broadening of the fluorescence profile. (a) Shows the integrated time resolved fluorescence with a mono-exponential decay and (b) the evolution of the FWHM of the spatial fluorescence measured at different times after excitation. During the first $5 \mathrm{~ns}$, there is less than $5 \mathrm{~nm}$ difference in the measured FWHM, slightly increasing at longer times. This increase could be due to the lower signal at longer times, or due to emission and reabsorption of photons from the initial excitation profile resulting in an actual broadening of the singlet distribution. From these measurements we estimate a precision of $10 \mathrm{~nm}$. 
SI 8- Fabrication and characterization of the tetracene crystals.

The tetracene crystals were prepared as follows: $1 \mathrm{mg}$ of tetracene (99.99\% Sigma-Aldrich) was added to $1 \mathrm{~mL}$ of toluene. The solution was stirred on a hotplate at a temperature of $60^{\circ} \mathrm{C}$ for 30 minutes, resulting in an oversaturated solution with a fraction of the tetracene undissolved in the toluene solution. The solution was cooled down slowly to $40{ }^{\circ} \mathrm{C}$ and filtered through a $0.22 \mu \mathrm{m}$ PTFE filter (PerkinElmer) into a glass vial. After 30 seconds the crystals were drop casted on the substrate. The entire process is performed inside a nitrogen glovebox. This method resulted in thin crystals (50-200 $\mathrm{nm}$ ) with relatively large lateral dimensions (up to $500 \mu \mathrm{m}$ ). The extinction and fluorescence spectra are shown below in Figure $\mathrm{S} 8$.



Figure S8: Typical extinction and fluorescence spectrums of a drop casted tetracene single crystal with an 150 nm thickness, prepared as described in the manuscript. The crystal has a strong anisotropy along two in-plane axes parallel to the substate. Extinction and fluorescence spectra were taken in a transmission microscope. For the extinction spectra, the sample was illuminated by a collimated broadband white light source (SLS201L, Thorlabs) that was polarized along either the fast or slow tetracene axis with a polarizing filter (LPVISA100-MP2, Thorlabs). The transmitted light was collected with a 60x objective (Nikon CFI S Plan Fluor ELWD, NA 0.7) and send to a spectrometer (Princeton Instruments SP2300) connected to a camera (Princeton Instruments ProEM:512). For the fluorescence images the sample was excited with a $450 \mathrm{~nm} \mathrm{CW}$ laser and the emission was collected through a polarizing filter and $500 \mathrm{~nm}$ long pass filter (FELH0500, Thorlabs). 
SI 9-Extended analysis of anisotropic triplet exciton diffusion in tetracene.
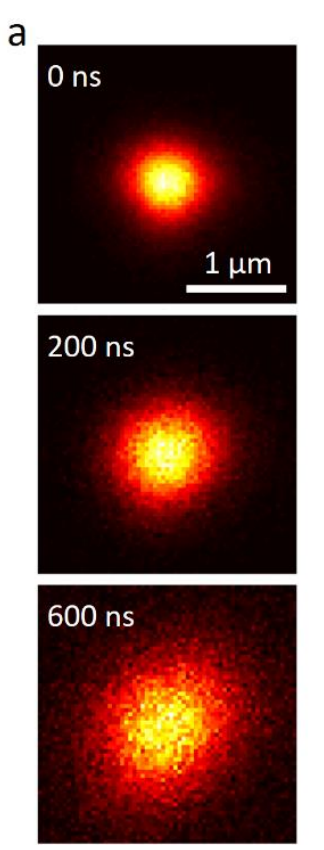
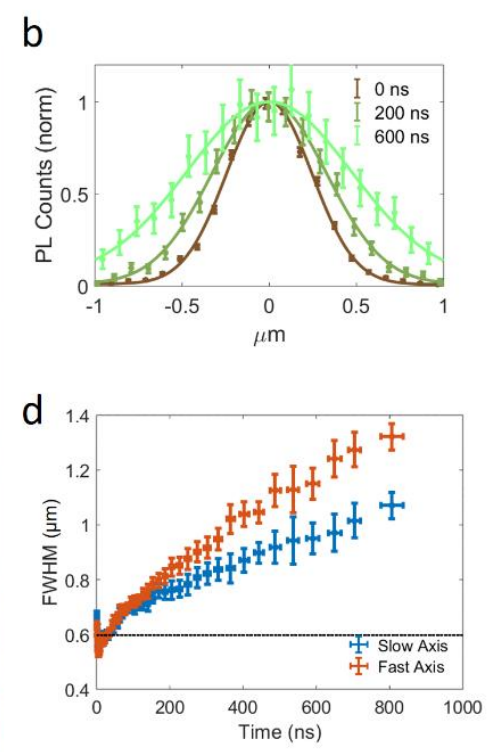

C
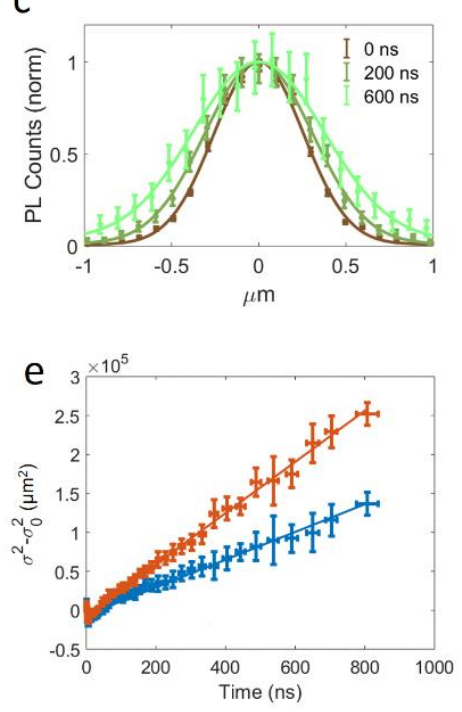

Figure S9: In the manuscript, we mentioned that the diffusion of triplet excitons in tetracene is anisotropic, but a complete analysis of this diffusion on the long time scales was not given. This figure shows the analysis of the diffusion measurements to obtain the diffusion coefficient of triplet excitons in the ab-plane of tetracene for an initial excitation density of $10^{18} \mathrm{~cm}^{-3}$. (a) Shows the images of the spatial distribution of the fluorescence for $t=0$ ns, 200 ns and 600 ns. (b) and (c) display the cross sections along the fast and slow axis in tetracene. The solid curves in these figures are Gaussian fits to the data. (d) and (e) show the width of the spatial profile for the fast axis (orange) and slow axis (blue). The solid lines in (e) are fits with the function: $\sigma(t)^{2}-\sigma(0)^{2}=2 \mathrm{Dt}$. Where $\mathrm{t}$ is time and $\mathrm{D}$ the diffusion constant. $D$ is found to be $8.4 \times 10^{-4} \mathrm{~cm}^{2} \mathrm{~s}^{-1}$ for the fast axis and $1.6 \times 10^{-3} \mathrm{~cm}^{2} \mathrm{~s}^{-1}$ for the slow axis. These values and the ratio between the fast and slow diffusion are in the same order of magnitude as those found by Akselrod et al. $\left(2.28 \times 10^{-3} \mathrm{~cm}^{2} \mathrm{~s}^{-1}\right.$ and $1.35 \times 10^{-3} \mathrm{~cm}^{2} \mathrm{~s}^{-1}$ for the fast and slow axis, respectively). It should be noted that the anisotropic exciton diffusion becomes clear only after $100 \mathrm{~ns}$. Before that time the spatial profile of the fluorescence is dominated by other processes such as exciton-exciton annihilation. 

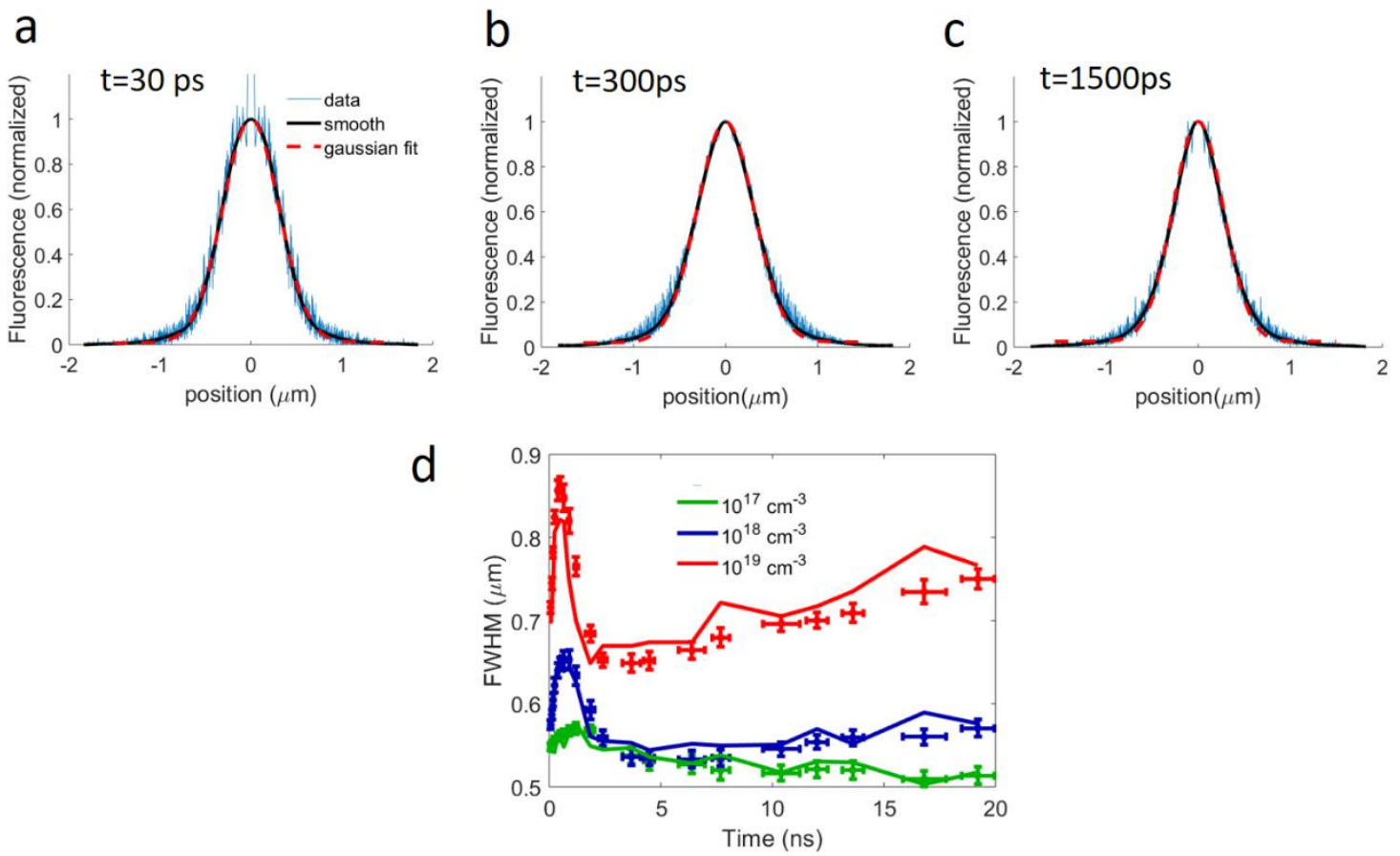

Figure S10: A diffraction limited excitation spot can be approximated by a Gaussian profile. In the linear regime, diffusion leads to a broadening of the profile, while maintaining the Gaussian shape. The diffusion constant can be obtained from the time evolution of the variance of the Gaussian profile. However, in case of non-linear effects, such as exciton-exciton annihilation, the shape of the excitation profile will deviate from a Gaussian. To test how this deviation may affect our measurements, we have compared the FWHM of the fluorescence spot as obtained from fitting a Gaussian to the data and by determining the FWHM of the data.

This second method consists of integrating the fluorescence for each distance from the center of the spot. The normalized fluorescence is plotted with the blue curve for three different times after excitation in (a-c) for the initial excitation of $10^{19}$ excitons $/ \mathrm{cm}^{3}$ (the data is mirrored across the $y$-axis, for better visualization of the shape). This normalized integrated fluorescence as a function of position is averaged using the MATLAB rloess tool (black curves). We also plotted in (a-c) a Gaussian fit to the data with the red dashed curve. We can see that even for the highest initial excitation density, where the largest exciton-exciton annihilation is expected, the Gaussian fit and the averaged data overlap. In (d), we have plotted with the circles the FWHM extracted from the Gaussians and with the solid lines the FWHM extracted from the averaged data, showing a very similar trend. Therefore, we can conclude that both methods give equivalent results. 
In this section, we calculate first analytically the minimum theoretical achievable spatial profile of delayed singlets due to SF followed by TTA in an idealized SF/TTA material. We show that the spatial profile of the delayed singlets in this ideal material is proportional to the excitation profile squared $\left(C * N_{S p}(0)^{2}\right)$. Since the excitation profile is approximated as a Gaussian, this leads to a profile for the delayed singlets with $\sigma_{\text {excitation }} / \sqrt{2}$.

The ideal material is described by Eqs. 2-4 in the manuscript and, on top of that, we assume zero diffusion $\left(D_{T}=0\right.$ and $\left.D_{s}=0\right)$. We investigate the time scale when $N_{s p}>>N_{s d}$ (which is reasonable in the first nanosecond after excitation). We work at low excitation density, such that singlet-singlet annihilation may be ignored. For simplicity, we disregard the radiative decay, but any rate would not modify the result. These assumptions simplify Eqs. 2-4 of the manuscript further to:

$$
\begin{gathered}
\frac{d N_{\mathrm{Sp}}}{d t}=-k_{\mathrm{fis}} N_{\mathrm{Sp}} \\
\frac{d N_{T}}{d t}=-2 k_{\mathrm{fus}} N_{\mathrm{T}}^{2}+k_{\mathrm{fis}} N_{\mathrm{Sp}} \\
\frac{d N_{\mathrm{Sd}}}{d t}=k_{\mathrm{fus}} N_{\mathrm{T}}^{2}-k_{\mathrm{fis}} N_{\mathrm{Sd}}
\end{gathered}
$$

and therefore:

$$
N_{\mathrm{Sp}}(t)=N_{\mathrm{Sp}}(0) e^{-\mathrm{k}_{\mathrm{fis}} t}
$$

We let the ideal material have the property that at short times

$$
\int k_{\mathrm{fus}} N_{\mathrm{T}}^{2} d t \ll \int k_{\mathrm{fis}} N_{\mathrm{S}} d t
$$

which is satisfied at very low excitation densities or in materials where the fission rate is much larger than the fusion rate. We then have

$$
N_{\mathrm{T}}(t)=\int_{0}^{t} k_{\mathrm{fis}} N_{\mathrm{Sp}}(t) d t=\left(1-e^{-k_{\mathrm{fis}} t}\right) N_{\mathrm{Sp}}(0)=f(t) N_{\mathrm{Sp}}(0),
$$

leading to

$$
\begin{gathered}
\frac{d N_{\mathrm{Sd}}}{d t}=f(t)^{2} N_{\mathrm{Sp}}(0)^{2}-k_{\mathrm{fis}} N_{\mathrm{Sd}}(t), \\
N_{\mathrm{Sd}}(t)=\left[\int_{0}^{t} f(\tau)^{2} e^{-k_{\mathrm{fis}}(t-\tau)} d \tau\right] N_{\mathrm{Sp}}(0)^{2}
\end{gathered}
$$

Which shows that the delayed singlets have the same profile as the prompt singlets squared.

To illustrate this result, we simulated the spatial profiles with the model described in Fig. 2 of the manuscript and with parameters: $\mathrm{k}_{\mathrm{fis}}=5 \mathrm{~ns}^{-1}, \mathrm{k}_{\mathrm{fus}}=10^{-19} \mathrm{~cm}^{3} \mathrm{~ns}^{-1}$ and $\mathrm{N}_{0}=10^{17} \mathrm{~cm}^{-3}$ and all other parameters set to 0 , as plotted in Fig. S11 

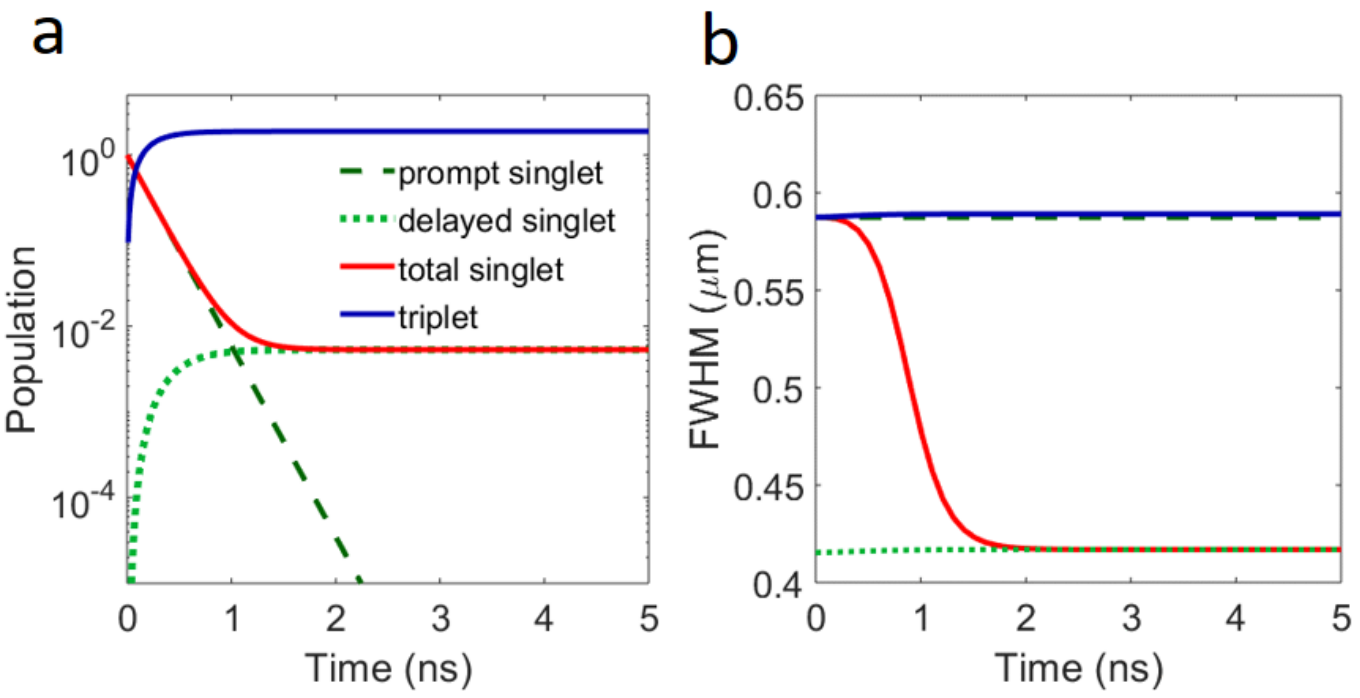

Figure S11: Simulation of the spatial evolution of the exciton profile in tetracene with $\mathrm{k}_{\mathrm{fis}}=5 \mathrm{~ns}^{-1}, \mathrm{k}_{\mathrm{fus}}=10^{-}$ ${ }^{19} \mathrm{~cm}^{3} \mathrm{~ns}^{-1}$ and $\mathrm{N}_{0}=10^{17} \mathrm{~cm}^{-3}$, and all the other parameters set to 0 . In (b) can be seen that the size of the singlet spot decreases from $590 \mathrm{~nm}$ to $417 \mathrm{~nm}$, which equals a decrease by a factor of $\sqrt{2}$.

SI 12 -Spatial transient Absorption measurements in tetracene.

a
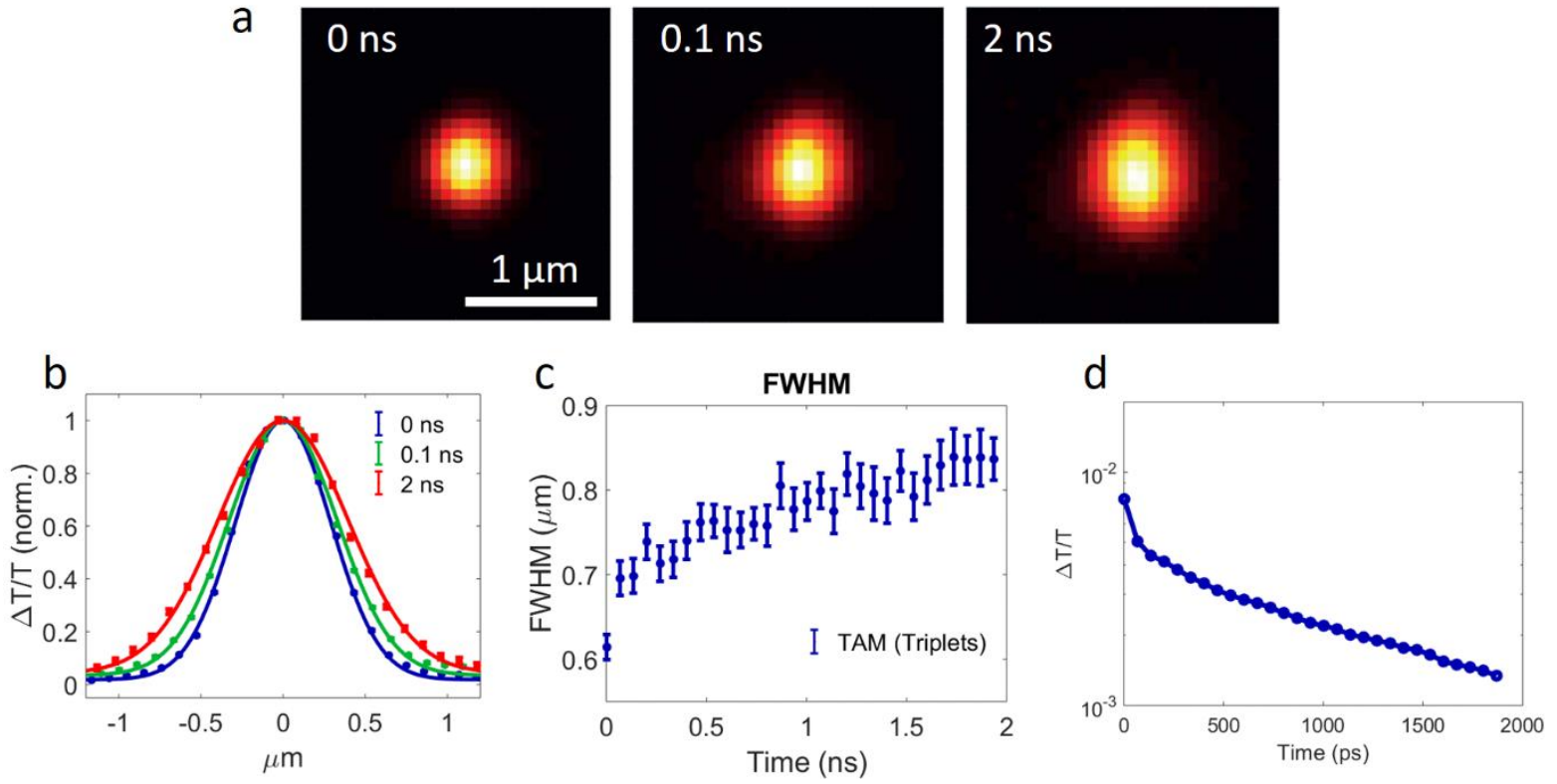

Figure S12: Transient absorption microscopy measurements for an excitation density of $10^{19} \mathrm{~cm}^{-3}$. (a) Shows the broadening of the transient absorption maps for three different pump-probe delay times. (b) Shows the horizontal cross sections of the spots of (a) and the fits with a Gaussian profile (solid curves). (c) is the evolution of the FWHM, obtained from the Gaussian fit of the cross sections, as plotted in Fig. 3 of the manuscript. The width of the cross section increases over time, in contrast to the fluorescence measurements for the same excitation intensity. (d) shows the differential transmission at the maximum of the signal as a function of time, displaying a multi-component decay. 
SI 13 - Intensity dependent measurements of tetracene with simplified model.

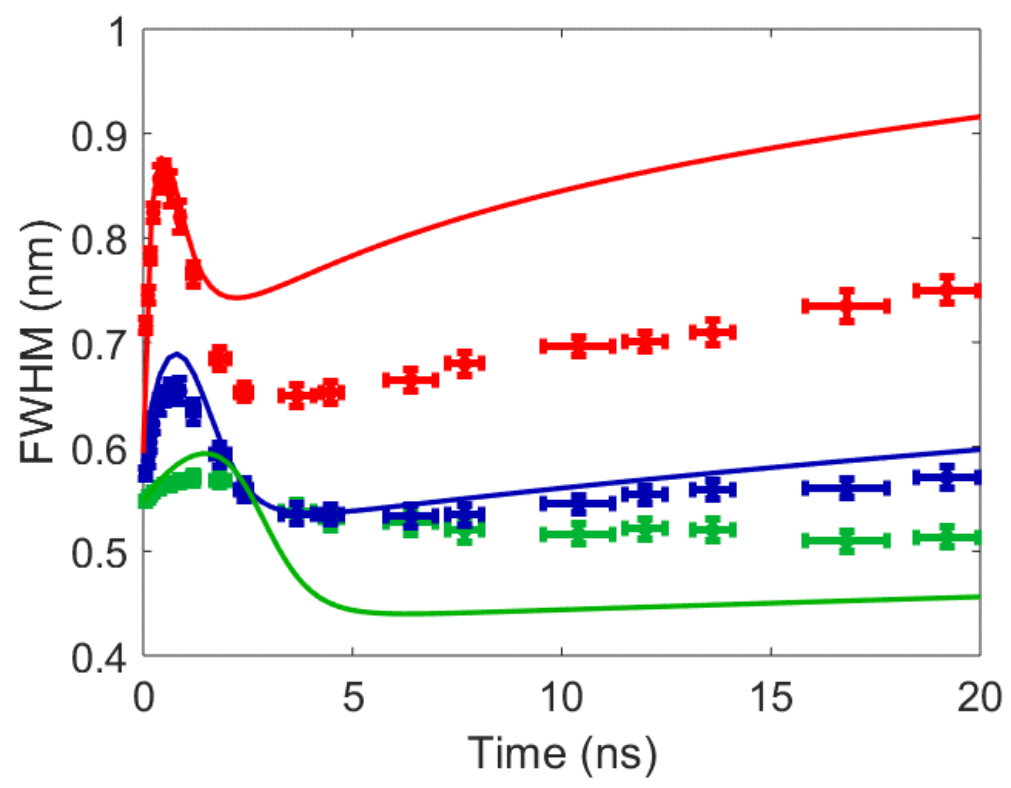

Figure S13: As discussed in the manuscript, we have attempted to fit the evolution of the fluorescence profiles with a model consisting of only triplet and singlet states (i.e., neglecting triplet pairs). This model and the fits are described in Fig $2 a$ and equations 2-4 of the manuscript. Here, the fits to the data with an initial excitation density of $10^{17}$ (green) $10^{18}$ (blue) $\mathrm{cm}^{-3}$ and $10^{19}$ (red) are shown. A single set of parameters is used to fit all the profile evolutions. Clearly, this model could not capture the laser intensity dependence of the spatial evolution of the FWHM of the fluorescence, as explained in the manuscript. The parameters for the fits shown with the solid lines, are given in table 2 of the manuscript. 
SI 14-Differential equation for the simulation of the effective negative exciton diffusion

The three differential equations below are used to model the evolution of the spatial distribution of the excitons as shown in Fig. 4(a) and (b) of the manuscript. The simulation is done for an initial Gaussian excitation profile and excitation densities of $10^{17}, 10^{18}$ and $10^{19} \mathrm{~cm}^{3} \mathrm{~ns}^{-1}$ using an Euler algorithm with 1 ps time step. The same set of parameters is used to simultaneously fit the spatial evolution of the triplet and the singlet states and at all the excitation densities. The result is plotted with the curves shown in Fig. $4 \mathrm{c}$ of the manuscript. All the variables are fitting parameters, except for the radiative decay rate, which is taken from literature ${ }^{2,6,7}$. The values that we obtain from the fits are given in table S14 and in agreement with earlier reported values ${ }^{2,6-8}$.

$$
\begin{gathered}
\frac{d N_{S}}{d t}=-\left(k_{S}+k_{f i s}\right) N_{S}-k_{S S A} N_{S}^{2}+k_{f u s} N_{\text {TTpair }}+k_{T T A} N_{T}^{2}+\nabla \cdot\left(D_{S} \nabla N_{S}\right), \\
\frac{d N_{\text {TTpair }}}{d t}=-\left(k_{\text {TTpair }}+k_{\text {fus }}+k_{\text {disS }}\right) N_{\text {TTpair }}+k_{f i s} N_{S}+k_{\text {merge }} N_{T}+\nabla \cdot\left(D_{T} \nabla N_{T T p a i r}\right), \\
\frac{d N_{T}}{d t}=-k_{T} N_{T}-2 k_{\text {merge }} N_{T}^{2}+2 k_{\text {diss }} N_{\text {TTpair }}-2 k_{T T A} N_{T}^{2}+\nabla \cdot\left(D_{T} \nabla N_{T}\right), \\
D_{S}=\left(\begin{array}{cc}
D_{S x} & 0 \\
0 & D_{S y}
\end{array}\right) \quad D_{T}=\left(\begin{array}{cc}
D_{T x} & 0 \\
0 & D_{T y}
\end{array}\right)
\end{gathered}
$$

Table S14: Simulation parameters for the extended model corresponding to Fig. 4 of the main

\begin{tabular}{cll} 
VARIABLE & MEANING & RATE \\
\hline$k_{S}$ & Radiative decay & $0.08 \mathrm{~ns}^{-1}$ \\
$k_{T T \text { pair }}$ & Triplet pair decay & $0.02 \mathrm{~ns}^{-1}$ \\
$k_{T}$ & Triplet decay & $210^{-3} \mathrm{~ns}^{-1}$ \\
$k_{S S A}$ & Singlet-singlet annihilation & $410^{-18} \mathrm{~cm}^{3} \mathrm{~ns}^{-1}$ \\
$k_{T T A}$ & Triplet-triplet annihilation (to singlet) & $410^{-20} \mathrm{~cm}^{3} \mathrm{~ns}^{-1}$ \\
$k_{\text {fis }}$ & Singlet to triplet pair & $0.5 \mathrm{~ns}^{-1}$ \\
$k_{f u s}$ & Triplet pair to singlet & $3 \mathrm{~ns}^{-1}$ \\
$k_{\text {diss }}$ & Triplet pair to triplet & $1.5 \mathrm{~ns}^{-1}$ \\
$k_{\text {merge }}$ & Triplet to triplet pair & $0.015 \mathrm{~ns}^{-1}$ \\
$D_{T x}, D_{T y}$ & Triplet diffusion slow, fast & $810^{-4}, 1.610^{-3} \mathrm{~cm}^{2} \mathrm{~s}^{-1}$ \\
$D_{S x}, D_{S y}$ & Singlet diffusion slow, fast & $0.03 \mathrm{~cm}^{2} \mathrm{~s}^{-1}, 0.03 \mathrm{~cm}^{2} \mathrm{~s}^{-1}$
\end{tabular}

manuscript. 
SI 15-Measurement and simulation of time resolved fluorescent decay.
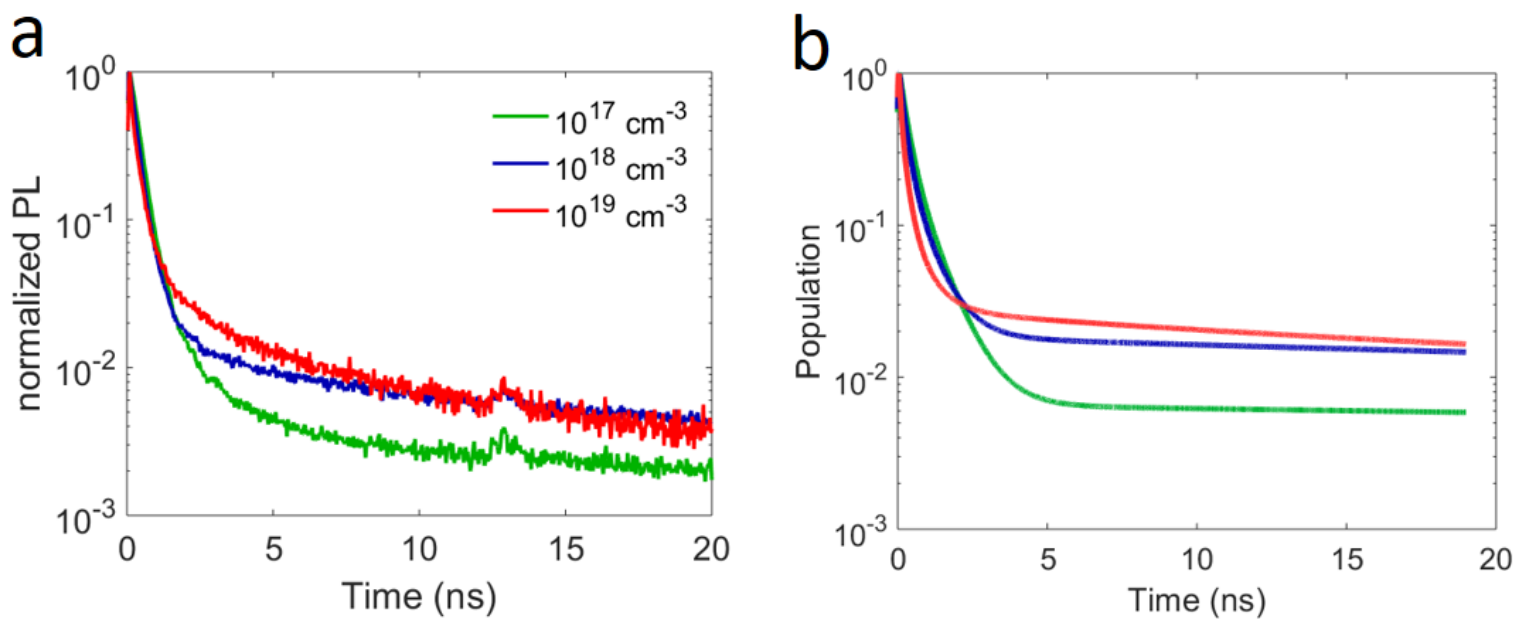

Figure S15: (a) Shows the measured fluorescence decay for the first $20 \mathrm{~ns}$, integrated over the entire excitation spot for three different excitation powers, as indicated in the legend. There is a slight bump in the measurements around $12.5 \mathrm{~ns}$, which originates from the $80 \mathrm{MHz}$ laser oscillator which is not filtered out perfectly by the pulse picker. (b) Shows the simulated decay of the tetracene PL. These simulations are obtained by running the three-state kinetic model, described in Fig. 4 of the manuscript and SI Table 11. The experimental and simulated curves show a very similar laser power dependence, confirming that the simulation captures the dynamics of the system reasonably well. The variables used in the simulation are given in table 3 of the manuscript. It should be noted that these parameters are obtained by fitting the evolution of the FWHM of the fluorescence profile curves and are not a fit to the decay curves. 
References

(1) Zhang, B.; Zhang, C.; Xu, Y.; Wang, R.; He, B.; Liu, Y.; Zhang, S.; Wang, X.; Xiao, M. Polarization-Dependent Exciton Dynamics in Tetracene Single Crystals. J. Chem. Phys. 2014, 141 (24), 244303. https://doi.org/10.1063/1.4904385.

(2) Wan, Y.; Guo, Z.; Zhu, T.; Yan, S.; Johnson, J.; Huang, L. Cooperative Singlet and Triplet Exciton Transport in Tetracene Crystals Visualized by Ultrafast Microscopy. Nat. Chem. 2015, 7 (10), 785-792. https://doi.org/10.1038/nchem.2348.

(3) Saad, N. A.; Rao, D. N.; Naraharisetty, S. R. G.; Sethupathy, M.; Sahoo, C. Ultrafast Pump-Probe Signal Detection Using a Data Acquisition Card. J. Instrum. 2018, 13 (10), P10027. https://doi.org/10.1088/1748-0221/13/10/p10027.

(4) Werley, C. A.; Teo, S. M.; Nelson, K. A. Pulsed Laser Noise Analysis and Pump-Probe Signal Detection with a Data Acquisition Card. Rev. Sci. Instrum. 2011, 82 (12), 123108. https://doi.org/10.1063/1.3669783.

(5) Akselrod, G. M.; Deotare, P. B.; Thompson, N. J.; Lee, J.; Tisdale, W. A.; Baldo, M. A.; Menon, V. M.; Bulovic, V. Visualization of Exciton Transport in Ordered and Disordered Molecular Solids. Nat. Commun. 2014, 5, 3646. https://doi.org/10.1038/ncomms4646.

(6) Piland, G. B.; Bardeen, C. J. How Morphology Affects Singlet Fission in Crystalline Tetracene. J. Phys. Chem. Lett. 2015, 6 (10), 1841-1846. https://doi.org/10.1021/acs.jpclett.5b00569.

(7) Wilson, M. W. B.; Rao, A.; Johnson, K.; Gélinas, S.; Di Pietro, R.; Clark, J.; Friend, R. $\mathrm{H}$. Temperature-Independent Singlet Exciton Fission in Tetracene. J. Am. Chem. Soc. 2013, 135 (44), 16680-16688. https://doi.org/10.1021/ja408854u.

(8) Burdett, J. J.; Bardeen, C. J. The Dynamics of Singlet Fission in Crystalline Tetracene and Covalent Analogs. Acc. Chem. Res. 2013, 46 (6), 1312-1320. https://doi.org/10.1021/ar300191w. 